\title{
HALL SUBGROUPS AND $p$-SOLVABILITY
}

\author{
A. GONCALVES AND C. Y. HO
}

ABSTRACT. Let $G$ be a finite group and let $\pi(G)=\left\{p, q_{1}, \ldots, q_{r}\right\}$ be the set of all prime divisors of $G$. Suppose there is a $p^{\prime}$-Hall subgroup $H$. If there are subgroups $P, Q_{1}, \ldots, Q_{r}$ such that $P \in \operatorname{Syl}_{p}(G), Q_{i} \in \operatorname{Syl}_{q_{i}}(H)$, and $L_{i}=P Q_{i}$ is a subgroup, $i=1, \ldots, r$, then $G$ is $p$-solvable. Moreover, if the subgroup $H$ is solvable, then $G$ is solvable too.

1. Introduction. In this paper, the following theorem will be proved.

Theorem. Suppose that $G$ is a finite group and $\pi(G)=\left\{p, q_{1}, \ldots, q_{r}\right\}$ is the set of all prime divisors of $G$. If the following holds, then $G$ is p-solvable.

(a) There is a $p^{\prime}$-Hall subgroup $H$.

(b) There are subgroups $P, Q_{1} \ldots, Q_{r}$ such that $P \in \operatorname{Syl}_{p}(G), Q_{i} \in$ $\operatorname{Syl}_{q_{i}}(H)$, and $L_{i}=P Q_{i}$ is a subgroup, $i=1, \ldots, r$.

Moreover, if the group $H$ in (a) is solvable, then $G$ is solvable too.

The group $X=\operatorname{PSL}(2,7)$ has subgroups $A, B, C$ where $A \in \operatorname{Syl}_{2}(X)$, $B \in \operatorname{Syl}_{3}(X), C \in \operatorname{Syl}_{7}(X)$ such that $A B, B C$ are subgroups of $X$. Of course $X$ is not $r$-solvable for $r=2,3$ or 7 .

Our notation follows Gorenstein [2]. We also let $\operatorname{Syl}_{q}(G)$ denote the set of Sylow $q$-subgroups of $G$. All groups considered in this paper are of finite order.

2. Proof of the Theorem. For completeness, we state the known result.

Lemma 1 (Glauberman). Let $G$ be a group with $O_{p}(G) \neq 1$ which is $p$ constrained and p-stable, $p$ odd. If $P \in \operatorname{Syl}_{p}(G)$, then $G=O_{p^{\prime}}(G) N_{G}(Z J(P))$. In particular, if $O_{p^{\prime}}(G)=1$, then $Z J(P) \triangleleft G$.

Proof. See [2, p. 279].

From now on we let $G$ be a minimal counterexample to the Theorem and use the notation $P, H, L_{i}, Q_{i}, i=1, \ldots, r$, which appeared in the statement of the Theorem. Since $H$ is $p$-solvable, $P$ does not normalize any nontrivial subgroup of $H$. Otherwise $G$ would contain a nontrivial $p$-solvable normal subgroup and by induction $G$ would not be a counterexample.

Lemma 2. If $H$ is solvable, then the theorem holds.

Proof. Since $H$ is solvable, $O_{q_{i}}(H) \neq 1$ for some $1 \leq i \leq r$. Also there

Received by the editors July 10,1974 .

AMS (MOS) subject classifications (1970). Primary 20F 15; Secondary $20 \mathrm{~F} 03$.

Key words and phrases. $p^{\prime}$-Hall subgroup, $Z(J(P)), O_{p}(G), p$-solvable, Syl ${ }_{p}(G)$. 
is a subgroup $C_{i}$ such that $H=Q_{i} C_{i}$. As $O_{q_{i}}(H) \subset Q_{i}$, we have

$$
\left(O_{q_{i}}(H)\right)^{G}=\left(O_{q_{i}}(H)\right)^{P H}=\left(O_{q_{i}}(H)\right)^{\left(P Q_{i}\right) C_{i}}=\left(O_{q_{i}}(H)\right)^{C{ }^{L} L_{i}}=O_{q_{i}}(H)^{L_{i}} \subset L_{i}
$$

Hence $G$ has a nontrivial normal solvable subgroup and the proof of the Theorem can be completed by induction.

Lemma 3. $P$ does not normalize any nontrivial $p^{\prime}$-subgroup.

Proof. Let $Q$ be any $p^{\prime}$-subgroup which is normalized by $P$. Set $R=$ $P \cdot Q$. Then $R=R \cap G=R \cap P \cdot H=P \cdot(R \cap H)$. Hence $R \cap H$ is a $p^{\prime}$-Hall subgroup of $R$. Since $Q=O_{p^{\prime}}(R), Q=R \cap H \subseteq H$. This implies that $P$ normalizes the subgroup $Q$ of $H$, which forces $Q=1$ as required.

Lemma 4. $O_{q_{i}}\left(L_{i}\right)=1, i=1, \ldots, r$.

Proof. Clear from Lemma 3.

Lemma 5. If $p \neq 3$, then the Theorem holds.

Proof. If $p=2$, then $|H|$ is odd. By the Feit-Thompson theorem [1], we see that $H$ is solvable. Lemma 2 implies the desired conclusion in this case.

Suppose $p \geq 5$. Then $L_{i}$ is $p$-constrained and $p$-stable [2, Theorem 5.1, p. 234]. By Lemmas 1 and 4 we see that $N_{G}\left(Z(J(P))\right.$ contains $Q_{i}, i=1, \ldots$, $r$. Hence $Z(J(P)) \triangleleft G$ which is impossible. This completes the proof of Lemma 5.

By Lemmas 5 and 2 we may assume $p=3$ and $|H|$ is even. Hence there is $j$ such that $q_{j}=2(1 \leq j \leq r)$. Let $K=\left\langle P, Q_{i} i \neq j\right\rangle$. Lemma 1 shows that $Z J(P) \triangleleft K$. Hence $N=O_{3}(K) \neq 1$. Since $|G: K|$ is a power of $2, G=K L_{j}$. Let $D=K \cap L_{j}$. Then $D \supseteq P \supseteq N$. Therefore

$$
N^{G}=N^{K L_{j}}=N^{L}{ }_{j} \subseteq D^{L_{j}} \subseteq L_{j}
$$

Since $L_{j}$ is solvable, $N^{G}$ is a nontrivial normal solvable subgroup of $G$ which is impossible. This completes the proof of the Theorem.

Remarks. (1) If the center of $P$ is weakly closed in $P$, then it can be shown, without using the $Z J$ theorem, that $G$ is $p$-solvable.

(2) The proof of the Theorem shows the following: Let $S$ be a set of Sylow subgroups of $H$ and $p \geq 5$. If $H=\langle Q \mid Q \in S\rangle$ and $L=P Q$ is a subgroup for any $q$ in $S$, then $G$ is $p$-solvable.

\section{REFERENCES}

1. W. Feit and J. G. Thompson, Solvability of groups of odd order, Pacific J. Math. 13 (1963), 775-1029. MR 29 \#3538.

2. D. Gorenstein, Finite groups, Harper \& Row, New York, 1968. MR 38 \#229.

DEPARTAMENTO DE MATEMATICA, UNIVERSIDADE DE BRASILIA, BRASILIA, BRASIL 